\title{
The small weight codewords of the functional codes associated to non-singular Hermitian varieties
}

\author{
F.A.B. Edoukou*, A. Hallez† F. Rodier and L. Storme \\ May 7, 2010
}

\begin{abstract}
This article studies the small weight codewords of the functional code $C_{H e r m}(\mathrm{X})$, with $\mathrm{X}$ a non-singular Hermitian variety of $\mathrm{PG}\left(N, q^{2}\right)$. The main result of this article is that the small weight codewords correspond to the intersections of $\mathrm{X}$ with the singular Hermitian varieties of $\mathrm{PG}\left(N, q^{2}\right)$ consisting of $q+1$ hyperplanes through a common $(N-2)$-dimensional space $\Pi$, forming a Baer subline in the quotient space of $\Pi$. The number of codewords having these small weights is also calculated. In this way, similar results are obtained to the functional codes $C_{2}(\mathrm{Q}), \mathrm{Q}$ a non-singular quadric [4], and $C_{2}(\mathrm{X}), \mathrm{X}$ a non-singular Hermitian variety [5].
\end{abstract}

Dedicated to the memory of András Gács (1969-2009)

\section{Introduction}

Consider a non-singular Hermitian variety $\mathrm{X}$ of $\mathrm{PG}\left(N, q^{2}\right)\left[7\right.$, Chapter 23]. Let $W_{i} \in$ $\operatorname{PG}\left(N, q^{2}\right)$ be the set of points with homogeneous coordinates $\left(X_{0}, \ldots, X_{N}\right)$ such that $X_{j}=0$ for $j<i$ and $X_{i} \neq 0$. The family $\left\{W_{i}\right\}_{0 \leq i \leq N}$ is a partition of $\operatorname{PG}\left(N, q^{2}\right)$. Let $\mathcal{F}$ be the $\mathbb{F}_{q}$-vector space of the zero polynomial and all homogeneous polynomials $\left(X_{0}, \ldots, X_{N}\right) A\left(X_{0}^{q}, \ldots, X_{N}^{q}\right)$ of degree $q+1$ in $N+1$ variables, with $A=\left(a_{i j}\right), 0 \leqslant i, j \leqslant N$, $a_{i j}^{q}=a_{j i}, a_{i j} \in \mathbb{F}_{q^{2}}$, defining Hermitian varieties of $\operatorname{PG}\left(N, q^{2}\right)$.

In this article, a Hermitian form will always denote a non-zero polynomial belonging to $\mathcal{F}$.

The functional codes $C_{\text {Herm }}(\mathrm{X})$ that are investigated in this article are inspired by the article of Lachaud [11] on linear codes defined on algebraic varieties. We denote by $\mathcal{F}_{h}$ the set of the homogeneous polynomials of degree $h$ over the finite field $\mathbb{F}_{q}$ in the variables $X_{0}, \ldots, X_{N}$. In general, for a fixed algebraic variety $\mathrm{X}$ in $\operatorname{PG}(N, q)$ with $n=\# \mathrm{X}\left(\mathbb{F}_{q}\right)$,

${ }^{*}$ The research of this author is supported by a MOE-AcRF Tier 2 Research Grant, Singapore (No. T206B2204).

${ }^{\dagger}$ The research of this author is supported by a research grant of the Research council of Ghent University. 
denote the point set of $\mathrm{X}$ by $\mathrm{X}=\left\{P_{1}, \ldots, P_{n}\right\}$, where the coordinates of the points $P_{i}$ are normalized with respect to the leftmost non-zero coordinate. Define the map

$$
c: \mathcal{F}_{h} \rightarrow \mathbb{F}_{q}^{n}: f \mapsto c(f)=\left(f\left(P_{1}\right), \ldots, f\left(P_{n}\right)\right),
$$

where

$$
f(Q)=f\left(X_{0}, \ldots, X_{N}\right) / X_{i}^{h}, \quad \text { with } \quad Q=\left(X_{0}, \ldots, X_{n}\right) \in W_{i} .
$$

The map $c$ is also linear over $\mathbb{F}_{q}$. The functional code

$$
C_{h}(\mathrm{X})=\left\{\left(f\left(P_{1}\right), \ldots, f\left(P_{n}\right)\right) \| f \in \mathcal{F}_{h}\right\}
$$

is the image of the linear map c. Thus, it is a linear subspace of $\mathbb{F}_{q}^{n}$ and therefore a linear code. We now construct a functional code over $\mathbb{F}_{q}$ defined by Hermitian forms.

The functional code $C_{H e r m}(\mathrm{X})$ is the linear code

$$
C_{H e r m}(\mathrm{X})=\left\{\left(f\left(P_{1}\right), \ldots, f\left(P_{n}\right)\right) \| f \in \mathcal{F}\right\}
$$

defined over $\mathbb{F}_{q}$. We stress that because of the normalization of the points $Q$, the value $f(Q)$ always belongs to the subfield $\mathbb{F}_{q}$ of $\mathbb{F}_{q^{2}}$.

This linear code $C_{\text {Herm }}(\mathrm{X})$ has length $n=|\mathrm{X}|$ and dimension $k=N^{2}+2 N$ over $\mathbb{F}_{q}$. This dimension is determined in the following way. First of all, since $c$ is a linear map, from the Dimension Theorem in Linear Algebra we have

$$
\operatorname{dim} C_{\text {Herm }}(\mathrm{X})=\operatorname{dim} \mathcal{F}-\operatorname{dim} \operatorname{ker} c .
$$

Secondly, a Hermitian variety in $\operatorname{PG}\left(N, q^{2}\right)$ is defined by an equation

$$
\sum_{i=0}^{N} \sum_{j=0}^{N} a_{i j} X_{i} X_{j}^{q}=0, \quad \text { where } \quad a_{i j}^{q}=a_{j i} .
$$

There are $\left((N+1)^{2}-(N+1)\right) / 2=\left(N^{2}+N\right) / 2$ elements $a_{i j}$, with $i<j$. They belong to $\mathbb{F}_{q^{2}}$, so they define an $\left(N^{2}+N\right)$-dimensional vector space over $\mathbb{F}_{q}$. The elements $a_{00}, \ldots, a_{N N}$ belong to $\mathbb{F}_{q}$, so they contribute additionally $N+1$ to this dimension. So $\mathcal{F}$, the vector space over $\mathbb{F}_{q}$ defined by all the Hermitian forms on $\operatorname{PG}\left(N, q^{2}\right)$, has dimension equal to $N^{2}+2 N+1$. It is a known fact that two distinct Hermitian varieties cannot have equations which are a scalar multiple of each other. So, since we take the intersection of all Hermitian varieties with X (i.e. $\operatorname{dim} \operatorname{ker} c=1$ ), the dimension of $C_{H e r m}(\mathrm{X})$ is $N^{2}+2 N$.

We stress that we investigate the properties of the code $C_{H e r m}(\mathrm{X})$, on the basis of its linearity properties over $\mathbb{F}_{q}$.

The third fundamental parameter of this linear code $C_{H e r m}(\mathrm{X})$ is its minimum distance $d_{\text {min }}$.

We determine the 4 smallest weights of $C_{H e r m}(\mathrm{X})$ via geometrical arguments (Tables 4 and 6). The small weight codewords of $C_{H e r m}(\mathrm{X})$ correspond to the Hermitian varieties $\mathrm{X}^{\prime}$ of $\mathrm{PG}\left(N, q^{2}\right)$ having the largest intersections with $\mathrm{X}$ since they have the largest numbers of zeros. 
In this article, a Baer subline always denotes a projective subline defined over $\mathbb{F}_{q}$.

The arguments presented in this article prove that these small weight codewords correspond to Hermitian varieties $\mathrm{X}^{\prime}$ which are the union of $q+1$ hyperplanes of $\mathrm{PG}\left(N, q^{2}\right)$ through a common $(N-2)$-dimensional space $\Pi$, defining a Baer subline in the quotient geometry of $\Pi$. There are 7 different possibilities for the intersection of such Hermitian varieties with a non-singular Hermitian variety X, but some of them imply the same intersection size. We determine in this way the 4 smallest weights of the functional code $C_{\text {Herm }}(\mathrm{X})$ (Tables 4 and 6 ).

We also determine the exact number of codewords having the 4 smallest weights. This leads to the following main result of this paper.

Theorem 1.1 Let $\mathrm{X}$ be a non-degenerate Hermitian variety in $\operatorname{PG}\left(N, q^{2}\right)$. The code $C_{\text {Herm }}(\mathrm{X})$ is an $\left[n, k, d_{\text {min }}\right]_{q}$-code where,

$$
\begin{aligned}
& n=\frac{\left(q^{N+1}+(-1)^{N}\right)\left(q^{N}+(-1)^{N+1}\right)}{q^{2}-1}, \quad k=N^{2}+2 N, \\
& d_{\text {min }}= \begin{cases}q^{2 N-1}-q^{2 N-2}-2 q^{N-1}, & \text { if } N \text { is even } \\
q^{2 N-1}-q^{2 N-2}-q^{N}+q^{N-1}, & \text { if } N \text { is odd. }\end{cases}
\end{aligned}
$$

The second and the third weights are $w_{2}=d_{\min }+q^{N-1}$ and $w_{3}=d_{\text {min }}+2 q^{N-1}$ for $N$ even, respectively $w_{2}=d_{\text {min }}+q^{N}-q^{N-1}$ and $w_{3}=d_{\text {min }}+q^{N}$ for $N$ odd. The fourth weight is $w_{4}=d_{\text {min }}+q^{N-1}(q+1)$, both for $N$ even and for $N$ odd.

The number of codewords of these weights are given in Table 4 for $N$ even, respectively in Table 6 for $N$ odd.

In this way, this article contributes to the study of small weight codewords in functional codes. In [1, 2], Edoukou investigated the functional codes arising from the intersections of quadrics with the non-singular Hermitian variety in $\mathrm{PG}\left(3, q^{2}\right)$ and $\mathrm{PG}\left(4, q^{2}\right)$, and the functional codes arising from the intersections of quadrics with the non-singular quadrics and the quadratic cone in PG(3,q). In [5], Hallez and Storme continued this study on the functional codes arising from the intersections of quadrics with the non-singular Hermitian variety in $\operatorname{PG}\left(N, q^{2}\right), N<O\left(q^{2}\right)$.

In a first article, the authors extended the results of Edoukou to the functional codes arising from non-singular quadrics in $\operatorname{PG}(N, q)$ [4]. Since Hermitian varieties, like quadrics, are sets of self-polar points in $\operatorname{PG}\left(N, q^{2}\right)$, it is natural to study their functional codes.

To conclude this introduction, the notation $\left\langle\Pi_{s_{1}}, \Pi_{s_{2}}\right\rangle$ will always denote the space generated by the two subspaces $\Pi_{s_{1}}$ and $\Pi_{s_{2}}$ of $\operatorname{PG}(N, q)$. Similarly, the notation $\left\langle\Pi_{s}, \mathrm{Q}\right\rangle$ denotes the smallest subspace of $\operatorname{PG}(N, q)$ containing the subspace $\Pi_{s}$ and the quadric Q. 


\section{Hermitian varieties in $\mathrm{PG}\left(N, q^{2}\right)$}

For the fundamental properties of Hermitian varieties, we refer to [7, Chapter 23]. We repeat the properties of importance for the arguments in this article.

The non-singular Hermitian variety $\mathrm{X}$ in $\mathrm{PG}\left(N, q^{2}\right)$ has standard equation

$$
X_{0}^{q+1}+\cdots+X_{N}^{q+1}=0 .
$$

A non-singular Hermitian variety in $\operatorname{PG}\left(N, q^{2}\right)$ contains $\frac{\left(q^{N+1}+(-1)^{N}\right)\left(q^{N}+(-1)^{N+1}\right)}{q^{2}-1}$ points, and the largest dimensional spaces contained in a non-singular Hermitian variety of $\operatorname{PG}\left(N, q^{2}\right)$ have dimension $\left\lfloor\frac{N-1}{2}\right\rfloor$, where $\lfloor x\rfloor$ denotes the largest integer smaller than or equal to $x$.

We denote by $\Pi_{d}$ a $d$-dimensional subspace of $\operatorname{PG}\left(N, q^{2}\right)$. All the Hermitian varieties of $\mathrm{PG}\left(N, q^{2}\right)$, including the non-singular Hermitian varieties, can be described as a Hermitian variety having a $d$-dimensional vertex $\Pi_{d}$ of singular points, $d \geq-1$, and having a nonsingular base $\mathrm{H}\left(N-d-1, q^{2}\right)$ in an $(N-d-1)$-dimensional space $\Pi_{N-d-1}$ skew to $\Pi_{d}$. In this article, the notation $\Pi_{d} \mathrm{H}\left(N-d-1, q^{2}\right)$ will always denote a Hermitian variety with vertex $\Pi_{d}$ and base the non-singular Hermitian variety $\mathrm{H}\left(N-d-1, q^{2}\right)$.

The largest dimensional subspaces contained in a Hermitian variety are called the generators of this Hermitian variety.

Since the sizes of (non-) singular Hermitian varieties of $\operatorname{PG}\left(N, q^{2}\right)$ are frequently used, they are explicitly listed.

In $\operatorname{PG}\left(N, q^{2}\right)$, a Hermitian variety having an $(N-d-1)$-dimensional vertex $\Pi_{N-d-1}$ and a non-singular Hermitian variety $\mathrm{H}\left(d, q^{2}\right)$ in $\mathrm{PG}\left(d, q^{2}\right)$ as base has the following size:

$$
\begin{aligned}
& d \text { even: } q^{2 N-1}+q^{2 N-3}+\cdots+q^{2 N-d+1}+q^{2 N-d-2}+q^{2 N-d-4}+\cdots+q^{2}+1 \\
& d \text { odd: } q^{2 N-1}+q^{2 N-3}+\cdots+q^{2 N-d}+q^{2 N-d-1}+q^{2 N-d-3}+\cdots+q^{2}+1
\end{aligned}
$$

Note that the size of the (non-)singular Hermitian variety having a non-singular Hermitian variety of odd dimension as base is always larger than the size of a (non-)singular Hermitian variety having a non-singular Hermitian variety of even dimension as base.

The Hermitian varieties having the largest size are the union of $q+1$ distinct hyperplanes of $\operatorname{PG}\left(N, q^{2}\right)$ and have size $q^{2 N-1}+q^{2 N-2}+q^{2 N-4}+q^{2 N-6}+\cdots+q^{2}+1$. The second largest Hermitian varieties in $\operatorname{PG}\left(N, q^{2}\right), N \geq 3$, are the Hermitian varieties having an $(N-4)$-dimensional vertex and a non-singular 3 -dimensional Hermitian variety as base. These Hermitian varieties have size $q^{2 N-1}+q^{2 N-3}+q^{2 N-4}+q^{2 N-6}+\cdots+q^{2}+1$. The third largest Hermitian variety in $\mathrm{PG}\left(N, q^{2}\right), N \geq 5$, has an $(N-6)$-dimensional vertex and a non-singular 5-dimensional Hermitian variety as base. These Hermitian varieties have size $q^{2 N-1}+q^{2 N-3}+q^{2 N-5}+q^{2 N-6}+q^{2 N-8}+\cdots+q^{2}+1$.

As mentioned in the introduction, the smallest weight codewords of the code $C_{H e r m}(\mathrm{X})$ correspond to the largest intersections of X with other Hermitian varieties $\mathrm{X}^{\prime}$ of $\mathrm{PG}\left(N, q^{2}\right)$. Let $V$ be the intersection of the Hermitian variety $\mathrm{X}$ with the Hermitian variety $\mathrm{X}^{\prime}$. The Hermitian pencil defined by $\mathrm{X}$ and $\mathrm{X}^{\prime}$ is the set of all the Hermitian varieties with equation $\lambda \mathrm{X}+\mu \mathrm{X}^{\prime},(\lambda, \mu) \in \mathbb{F}_{q}^{2} \backslash\{(0,0)\}$. Two distinct Hermitian varieties $\mathrm{X}$ and $\mathrm{X}^{\prime}$ define a unique 
pencil of Hermitian varieties. The points of $V=\mathrm{X} \cap \mathrm{X}^{\prime}$ belong to all the $q+1$ Hermitian varieties in this pencil, while any point of $\mathrm{PG}\left(N, q^{2}\right)$ not in $V$ belongs to exactly one of the Hermitian varieties in this pencil.

The fact that the Hermitian pencil $\lambda \mathrm{X}+\mu \mathrm{X}^{\prime}$ of two Hermitian varieties $\mathrm{X}$ and $\mathrm{X}^{\prime}$ is only defined for coefficients $(\lambda, \mu) \in \mathbb{F}_{q}^{2}$, was one of the reasons why in the introduction, the code ${ }_{\text {Herm }}(\mathrm{X})$ was defined over $\mathbb{F}_{q}$ instead of over $\mathbb{F}_{q^{2}}$.

Let $V=\mathrm{X} \cap \mathrm{X}^{\prime}$. The sum of the numbers of points in the $q+1$ Hermitian varieties of the pencil defined by $\mathrm{X}$ and $\mathrm{X}^{\prime}$ is $\left|\mathrm{PG}\left(N, q^{2}\right)\right|+q|V|$ points, since the points of $V$ lie in all the Hermitian varieties of the pencil and the other points of $\operatorname{PG}\left(N, q^{2}\right)$ lie in exactly one such Hermitian variety. So there is a Hermitian variety in the pencil containing at least $\left(\left|\mathrm{PG}\left(N, q^{2}\right)\right|+q|V|\right) /(q+1)$ points. Hence, a large intersection $V$ implies that there is a large Hermitian variety in the pencil of Hermitian varieties defined by $\mathrm{X}$ and $\mathrm{X}^{\prime}$.

If there is a Hermitian variety in the pencil of Hermitian varieties defined by $\mathrm{X}$ and $\mathrm{X}^{\prime}$, equal to the union of $q+1$ hyperplanes, then the desired conclusion is obtained that the largest intersections of $\mathrm{X}$ arise from the intersections of $\mathrm{X}$ with the Hermitian varieties which are the union of $q+1$ hyperplanes. So assume that all the $q+1$ Hermitian varieties in the pencil of Hermitian varieties defined by $\mathrm{X}$ and $\mathrm{X}^{\prime}$ are irreducible (i.e. there is not a Hermitian variety in this pencil which is the union of $q+1$ hyperplanes); this will lead to a contradiction, if $|V|$ is large enough. As already mentioned above, the largest irreducible Hermitian varieties in $\operatorname{PG}\left(N, q^{2}\right)$ are cones with vertex $\operatorname{PG}\left(N-4, q^{2}\right)$ and base $\mathrm{H}\left(3, q^{2}\right)$, a non-singular Hermitian variety in $\mathrm{PG}\left(3, q^{2}\right)$, and the second largest irreducible Hermitian varieties are cones with vertex $\mathrm{PG}\left(N-6, q^{2}\right)$ and base $\mathrm{H}\left(5, q^{2}\right)$, a non-singular Hermitian variety in $\mathrm{PG}\left(5, q^{2}\right)$. These observations will be used many times.

Remark 2.1 Consider a fixed line $T$ of $\mathrm{H}\left(3, q^{2}\right)$. Then the $q^{3}+q$ lines of $\mathrm{H}\left(3, q^{2}\right)$ intersecting $T$ in one point form a minimal cover of $\mathrm{H}\left(3, q^{2}\right)$. This cover is the smallest cover of $\mathrm{H}\left(3, q^{2}\right)[12]$.

There are exactly $(1+q)\left(q^{3}+1\right)$ such covers since this is the total number of lines of $\mathrm{H}\left(3, q^{2}\right)[7$, Table 23.1].

Theorem 2.2 In $\mathrm{PG}\left(N, q^{2}\right)$, with $N \geqslant 6$, if

$$
|V|>q^{2 N-2}+2 q^{2 N-4}+q^{2 N-5}+q^{2 N-6}+2 q^{2 N-7}+2 q^{2 N-9}+\cdots+2 q^{3}+q,
$$

then in the pencil of Hermitian varieties defined by $\mathrm{X}$ and $\mathrm{X}^{\prime}$, there is a Hermitian variety consisting of the union of $q+1$ hyperplanes.

Proof. Suppose that there is no Hermitian variety in the pencil of Hermitian varieties defined by $\mathrm{X}$ and $\mathrm{X}^{\prime}$ equal to the union of $q+1$ hyperplanes.

Since $|V|>q^{2 N-2}+q^{2 N-4}+2 q^{2 N-6}+q^{2 N-8}+\cdots+q^{2}+1$, the following inequality $\left(\left|\mathrm{PG}\left(N, q^{2}\right)\right|+q|V|\right) /(q+1)>\left|\Pi_{N-6} \mathrm{H}\left(5, q^{2}\right)\right|$ is valid; so there is a singular Hermitian variety $\Pi_{N-4} \mathrm{H}\left(3, q^{2}\right)$ in the pencil of Hermitian varieties defined by $\mathrm{X}$ and $\mathrm{X}^{\prime}$. With the lines of the cover of $\mathrm{H}\left(3, q^{2}\right)$ of Remark 2.1 , together with $\Pi_{N-4}, q^{3}+q$ different $(N-2)$ spaces $\Pi_{N-2}$ are formed. We wish to have that at least one of these $(N-2)$-dimensional spaces $\Pi_{N-2}$ intersects $\mathrm{X}$ in $q+1(N-3)$-spaces. All points of $V$ appear in at least 
one of these $\Pi_{N-2}$, so for at least one of these spaces, necessarily $\left|\Pi_{N-2} \cap V\right| \geqslant \frac{|V|}{q^{3}+q}$. If $\frac{|V|}{q^{3}+q}>\left|\Pi_{N-6} \mathrm{H}\left(3, q^{2}\right)\right|$, then $\Pi_{N-2} \cap \mathrm{X}$ is the union of $q+1(N-3)$-spaces. When $|V|>q^{2 N-2}+2 q^{2 N-4}+q^{2 N-5}+q^{2 N-6}+2 q^{2 N-7}+2 q^{2 N-9}+\cdots+2 q^{3}+q$, then this is valid. So $\Pi_{N-2} \cap \mathrm{X}=\bigcup_{i=1}^{q+1} \Pi_{N-3}^{(i)}$.

This means that $\mathrm{X}$ must have generators of dimension $N-3$.

\begin{tabular}{|c|c|c|}
\hline Hermitian variety & dimension generator & property fullfilled \\
\hline $\mathrm{X}=\mathrm{H}\left(2 N^{\prime}, q^{2}\right)$ & $N^{\prime}-1$ & $N^{\prime} \leqslant 2$ \\
\hline $\mathrm{X}=\mathrm{H}\left(2 N^{\prime}+1, q^{2}\right)$ & $N^{\prime}$ & $N^{\prime} \leqslant 2$ \\
\hline
\end{tabular}

Table 1: Non-discussed cases.

Except for the small cases for $N^{\prime}$, see Table 1, a contradiction is obtained, so there is a Hermitian variety consisting of the union of hyperplanes in the pencil of Hermitian varieties defined by $\mathrm{X}$ and $\mathrm{X}^{\prime}$.

The following results of Kestenband now are stated to compare the preceding bound on $|V|$ with the intersection size of two Hermitian varieties $\mathrm{X}$ and $\mathrm{X}^{\prime}$ in $\mathrm{PG}\left(N, q^{2}\right)$, where the pencil of Hermitian varieties defined by $\mathrm{X}$ and $\mathrm{X}^{\prime}$ does not contain a singular Hermitian variety which is the union of $q+1$ hyperplanes.

Theorem 2.3 (1) ([9, Lemma 2]) There exists a pencil of $q+1$ non-singular Hermitian varieties in $\mathrm{PG}\left(N=2 n, q^{2}\right)$ intersecting in

$$
\frac{\left(q^{2 n-1}-1\right)\left(q^{2 n+1}+1\right)}{q^{2}-1}=q^{4 n-2}+q^{4 n-4}+\cdots+q^{2}+1-q^{2 n-1}
$$

points.

(2) ([10, Lemma 3]) There exists a pencil of $q+1$ non-singular Hermitian varieties in $\mathrm{PG}\left(N=2 n-1, q^{2}\right)$ intersecting in

$$
\frac{\left(q^{2 n}-1\right)\left(q^{2 n-2}+1\right)}{q^{2}-1}=q^{4 n-4}+q^{4 n-6}+\cdots+q^{2 n+2}+q^{2 n}+2 q^{2 n-2}+q^{2 n-4}+\cdots+q^{2}+1
$$

points.

\section{Hermitian variety in $\mathrm{PG}\left(5, q^{2}\right)$}

This section now discusses the case that $\mathrm{X}$ is the Hermitian variety $\mathrm{H}\left(5, q^{2}\right)$ in 5 dimensions. Let $V$ be the intersection of $\mathrm{X}$ with another Hermitian variety $\mathrm{X}^{\prime}$ in $\mathrm{PG}\left(5, q^{2}\right)$.

If $|V|>q^{8}+q^{6}+2 q^{4}+q^{2}+1$, then $\left(\left|\mathrm{PG}\left(5, q^{2}\right)\right|+q|V|\right) /(q+1)>\left|\mathrm{H}\left(5, q^{2}\right)\right|$, so there is a cone $\Pi_{N-4} \mathrm{H}\left(3, q^{2}\right)=L \mathrm{H}\left(3, q^{2}\right)$ in the pencil of Hermitian varieties defined by $\mathrm{X}$ and $\mathrm{X}^{\prime}$, if it is assumed that no Hermitian variety in the pencil of Hermitian varieties defined by $\mathrm{X}$ and $\mathrm{X}^{\prime}$ is the union of $q+1$ hyperplanes. Form solids $\Pi_{3}^{(1)}, \ldots, \Pi_{3}^{\left(q^{3}+q\right)}$ with $L$ and the lines of a cover of $\mathrm{H}\left(3, q^{2}\right)$, as defined in Remark 2.1. If $|V|>q^{8}+2 q^{6}+q^{5}+q^{4}+2 q^{3}+q$, then there is a solid through $L$ intersecting $\mathrm{X}$ in $q+1$ planes. Now there are 3 different cases that need to be discussed: 
1. $L \subset V$,

2. $|L \cap V|=q+1$,

3. $|L \cap V|=1$.

Lemma 3.1 For $\mathrm{X}=\mathrm{H}\left(5, q^{2}\right)$, if $|V|>q^{8}+2 q^{6}+q^{5}+q^{4}+2 q^{3}+q$ and $L \subset V$, then there is a Hermitian variety consisting of the union of $q+1$ hyperplanes in the pencil of Hermitian varieties defined by $\mathrm{X}$ and $\mathrm{X}^{\prime}$.

Proof. Assume that no Hermitian variety in the pencil of Hermitian varieties defined by $\mathrm{X}$ and $\mathrm{X}^{\prime}$ is the union of $q+1$ hyperplanes. Since $\left(\left|\mathrm{PG}\left(5, q^{2}\right)\right|+q|V|\right) /(q+1)>\left|\mathrm{H}\left(5, q^{2}\right)\right|$, there is a singular Hermitian variety $L \mathrm{H}\left(3, q^{2}\right)$ in the pencil of Hermitian varieties defined by $\mathrm{X}$ and $\mathrm{X}^{\prime}$.

By Remark 2.1, $\mathrm{H}\left(3, q^{2}\right)$ can be covered by $q^{3}+q$ lines. Considering the $q^{3}+q$ solids defined by $L$ and the lines of this cover of $\mathrm{H}\left(3, q^{2}\right), L \mathrm{H}\left(3, q^{2}\right)$ is covered by $q^{3}+q$ solids. Since $|V| /\left(q^{3}+q\right)>\left|\mathrm{H}\left(3, q^{2}\right)\right|$, there is a solid $\Pi_{3}^{(1)}$ through $L$ intersecting $V$ in $q+1$ planes. Now $L$ lies in one of these planes, since $L \subset V$.

Every point of $V$ lies in at least one of these $q^{3}+q$ solids through $L$, defining the cover of $L \mathrm{H}\left(3, q^{2}\right)$.

In $\mathrm{H}\left(5, q^{2}\right)$, a line $L$ is contained in $q+1$ planes completely lying in $\mathrm{H}\left(5, q^{2}\right)$. Now we want to have a bound on $|V|$ so that we are sure that the line $L$ lies in more than $q+1$ planes contained in $\mathrm{H}\left(5, q^{2}\right)$. Because then a contradiction is obtained to our assumption that no Hermitian variety in the pencil of Hermitian varieties defined by $\mathrm{X}$ and $\mathrm{X}^{\prime}$ is the union of $q+1$ hyperplanes.

To find at least $q+2$ planes of $V$ through $L$, an inductive argument needs to be used stating that if $L$ lies in $x$ planes of $V$, then it lies in $x+1$ planes of $V$. To simplify the calculations, we describe how the existence of $q+1$ planes of $V$ through $L$ implies the existence of $q+2$ planes of $V$ through $L$, in case $|V|$ is large enough.

Assume that it is known that $q+1$ of the solids of the cover of size $q^{3}+q$ of $L \mathrm{H}\left(3, q^{2}\right)$ intersect $V$ in the union of $q+1$ planes, where these $q+1$ solids have distinct planes through $L$ in common with $V$. We want to have another solid which fullfils this condition, so that the desired contradiction is obtained.

The desired contradiction is obtained when

$$
|L|+\frac{|V|-(q+1)\left((q+1) q^{4}+q^{2}+1\right)}{q^{3}-1}>\left|\mathrm{H}\left(3, q^{2}\right)\right| .
$$

For the $q+1$ solids through $L$ intersecting $V$ in $q+1$ planes, each contain $(q+1) q^{4}+q^{2}+1$ points of $V$. Subtract this number from $|V|$. There remain $q^{3}-1$ solids for the cover of $L \mathrm{H}\left(3, q^{2}\right)$. So there is a solid containing at least

$$
|L|+\frac{|V|-(q+1)\left((q+1) q^{4}+q^{2}+1\right)}{q^{3}-1}>\left|\mathrm{H}\left(3, q^{2}\right)\right|
$$


points of $V$. Since the only Hermitian variety in $\mathrm{PG}\left(3, q^{2}\right)$ containing more than $\left|\mathrm{H}\left(3, q^{2}\right)\right|$ points consists of the union of $q+1$ planes, the desired $(q+2)$-th plane of $V$ through $L$ has been found.

The only problem that remains is that this $(q+2)$-th plane must be different from all the previous $q+1$ planes of $V$ through $L$. This goal is achieved in the following way. The cover of $\mathrm{H}\left(3, q^{2}\right)$ that is defined in Remark 2.1 consists of all the lines of $\mathrm{H}\left(3, q^{2}\right)$ intersecting a given line $T$ of $\mathrm{H}\left(3, q^{2}\right)$; this line $T$ not included. For finding the $(q+2)$-th plane of $V$ through $L$, select for the line $T$, which defines the cover of $\mathrm{H}\left(3, q^{2}\right)$, a line $T$ skew to the $q+1$ points of $\mathrm{H}\left(3, q^{2}\right)$ defining the $q+1$ planes of $V$ through $L$. This is possible since these $q+1$ points lie in total on at most $(q+1)^{2}$ lines of $\mathrm{H}\left(3, q^{2}\right)$. So there is certainly a line $T$ of $\mathrm{H}\left(3, q^{2}\right)$ skew to these $q+1$ points. Then we use the cover of $\mathrm{H}\left(3, q^{2}\right)$ of size $q^{3}+q$ defined by this line $T$. The particular property of the corresponding cover of $L \mathrm{H}\left(3, q^{2}\right)$ is that the $q+1$ planes of $V$ through $L$, already determined, lie in exactly one of those solids, so when we perform the division in the left hand side of (1), the $(q+2)$-th solid through $L$ intersecting $V$ in $q+1$ planes cannot contain one of the already determined $q+1$ planes of $V$ through $L$.

This gives at least $q+2$ planes of $\mathrm{H}\left(5, q^{2}\right)$ through $L$; which is impossible. So there is a Hermitian variety consisting of $q+1$ hyperplanes in the pencil of Hermitian varieties defined by $\mathrm{X}$ and $\mathrm{X}^{\prime}$. The condition in (1) is equivalent to

$$
|V|>q^{8}+2 q^{6}+q^{5}+q^{4}+q^{2}+q+1 .
$$

The most severe condition on $|V|$ arises from the fact that $|V| /\left(q^{3}+q\right)>\left|\mathrm{H}\left(3, q^{2}\right)\right|$; which implies $|V|>q^{8}+2 q^{6}+q^{5}+q^{4}+2 q^{3}+q$.

Lemma 3.2 For $\mathrm{X}=\mathrm{H}\left(5, q^{2}\right)$, if $|V|>q^{8}+4 q^{6}+q^{5}-3 q^{4}+4 q^{3}+3 q^{2}+q-1$ and $|L \cap V|=q+1$, then there is a Hermitian variety consisting of $q+1$ hyperplanes in the pencil of Hermitian varieties defined by $\mathrm{X}$ and $\mathrm{X}^{\prime}$.

Proof. Assume that no Hermitian variety in this pencil is the union of $q+1$ hyperplanes. Then, since the lower bound on $|V|$ of the beginning of this section is valid, there is a cone $L \mathrm{H}\left(3, q^{2}\right)$ in the pencil of Hermitian varieties defined by $\mathrm{X}$ and $\mathrm{X}^{\prime}$. Assume that $L \cap V=\left\{R_{1}, \ldots, R_{q+1}\right\}$. Let the polar space of the secant line $L$ with respect to $\mathrm{X}=$ $\mathrm{H}\left(5, q^{2}\right)$ be the 3 -dimensional space intersecting $\mathrm{H}\left(5, q^{2}\right)$ in the non-singular Hermitian variety $\mathrm{H}\left(3, q^{2}\right)_{L}$.

Suppose that we are sure that $x+1$ lines of a cover of size $q^{3}+q$ on $\mathrm{H}\left(3, q^{2}\right)$, as defined in Remark 2.1, define solids through $L$ intersecting $\mathrm{H}\left(5, q^{2}\right)$ in a union of $q+1$ planes. We are sure of this when

$$
q+1+\frac{|V|-x\left((q+1) q^{4}+q^{2}+1\right)}{q^{3}+q-x}>\left|\mathrm{H}\left(3, q^{2}\right)\right| .
$$

This is equivalent to $|V|>q^{8}+2 q^{6}+q^{5}+q^{3}-q^{2}+x\left(q^{4}-q^{3}+q+1\right)$.

Consider all covers of size $q^{3}+q$ on $\mathrm{H}\left(3, q^{2}\right)$ defined by Remark 2.1. There are exactly $(1+q)\left(q^{3}+1\right)$ such covers. Then we get at least $(1+q)\left(q^{3}+1\right)(x+1)$ lines of $\mathrm{H}\left(3, q^{2}\right)$ 
defining solids of $L \mathrm{H}\left(3, q^{2}\right)$ through $L$ intersecting $V$ in $q+1$ planes. But every such line could be counted up to $q^{3}+q$ times. Nevertheless, we get at least

$$
\frac{(1+q)\left(q^{3}+1\right)(x+1)}{q^{3}+q}>q(x+1)
$$

distinct lines of $\mathrm{H}\left(3, q^{2}\right)$ defining solids of $L \mathrm{H}\left(3, q^{2}\right)$ through $L$ intersecting $V$ in $q+1$ planes.

But then for more than $q(x+1)$ lines $\ell$ of the base $\mathrm{H}\left(3, q^{2}\right)$, it is known that the solid $\langle L, \ell\rangle$ contains a plane of $\mathrm{H}\left(3, q^{2}\right)$ through $R_{1}, \ldots, R_{q+1}$. So $R_{1}$ lies in planes contained in the intersection $V$. These planes lie in $T_{R_{1}}(\mathrm{X})=\left\langle R_{1}, \mathrm{H}\left(3, q^{2}\right)_{L}\right\rangle$, where $\left\langle R_{1}, \mathrm{H}\left(3, q^{2}\right)_{L}\right\rangle$ denotes the 4-dimensional space spanned by $R_{1}$ and the 3-dimensional Hermitian variety $\mathrm{H}\left(3, q^{2}\right)_{L}$. We prove that the cones $R_{i} \mathrm{H}\left(3, q^{2}\right)_{L}, i=1, \ldots, q+1$, lie completely in the intersection $V$ if $x$ is large enough.

Consider again the cone $L \mathrm{H}\left(3, q^{2}\right)$ in the pencil of Hermitian varieties defined by $\mathrm{X}$ and $\mathrm{X}^{\prime}$. Let $\ell$ be a line of the base $\mathrm{H}\left(3, q^{2}\right)$ defining a solid $\langle L, \ell\rangle$ intersecting $V$ in the union of $q+1$ planes, which pass one by one through $R_{1}, \ldots, R_{q+1}$. Then these $q+1$ planes intersect in a line $\ell^{\prime}$ lying on $\mathrm{H}\left(3, q^{2}\right)_{L}$. This line $\ell^{\prime}$ is skew to $L$, so determines $\langle L, \ell\rangle$ uniquely. Hence, different lines $\ell$ of $\mathrm{H}\left(3, q^{2}\right)$ define different lines $\ell^{\prime}$ of $\mathrm{H}\left(3, q^{2}\right)_{L}$.

So, we find more than $q(x+1)$ lines of $\mathrm{H}\left(3, q^{2}\right)_{L}$ completely lying in $V$. We can now prove that the cones $R_{i} \mathrm{H}\left(3, q^{2}\right)_{L}, i=1, \ldots, q+1$, lie completely on $V$.

Consider a point $P$ of the base $\mathrm{H}\left(3, q^{2}\right)_{L}$ and assume that $P$ does not lie on one of these $q(x+1)$ lines $\ell^{\prime}$ of $\mathrm{H}\left(3, q^{2}\right)_{L}$ lying in $V$. Then they all intersect $T_{P}\left(\mathrm{H}\left(3, q^{2}\right)_{L}\right)$ in a point. If $q(x+1)>2(q+1) q^{2}$, there is a point of $\mathrm{H}\left(3, q^{2}\right)_{L}$ in $T_{P}\left(\mathrm{H}\left(3, q^{2}\right)_{L}\right)$ on at least 3 of those lines. Denote this point by $S$ and these three lines by $\ell_{1}, \ell_{2}, \ell_{3}$. Then the three planes $\left\langle R_{i}, \ell_{1}\right\rangle,\left\langle R_{i}, \ell_{2}\right\rangle,\left\langle R_{i}, \ell_{3}\right\rangle$ lie completely in $V$. Then $T_{S}\left(\mathrm{H}\left(3, q^{2}\right)_{L}\right)$ shares already 3 lines with the intersection $V$, so it intersects $V$ in all $q+1$ lines $\ell_{j}, j=1, \ldots, q+1$, of $\mathrm{H}\left(3, q^{2}\right)_{L}$ through $S$, and similarly, all $q+1$ planes $\left\langle R_{i}, \ell_{j}\right\rangle, j=1, \ldots, q+1$, lie completely in $V$. But one of these lines $\ell_{j}$ is the line $S P$, so the line $R_{i} P$ belongs to the intersection $V$. So every point of the cone $R_{i} \mathrm{H}\left(3, q^{2}\right)_{L}$ lies in $V$.

The tangent cones $R_{i} \mathrm{H}\left(3, q^{2}\right)_{L}$ to $\mathrm{H}\left(5, q^{2}\right)$ lie in $q+1$ hyperplanes through the polar space $\Pi_{3}$ of $L$ with respect to $\mathrm{X}$, and these $q+1$ hyperplanes define a Hermitian variety $\mathrm{X}^{\prime \prime}$. Let $S$ be a point of $\Pi_{3} \backslash \mathrm{H}\left(3, q^{2}\right)_{L}$. There is a unique Hermitian variety $\mathrm{X}^{\prime \prime \prime}$, containing $S$, in the pencil of Hermitian varieties defined by $\mathrm{X}$ and $\mathrm{X}^{\prime}$. This Hermitian variety must be the union of the $q+1$ hyperplanes $\left\langle R_{i}, \mathrm{H}\left(3, q^{2}\right)_{L}\right\rangle$, but then we find that the pencil of Hermitian varieties defined by $\mathrm{X}$ and $\mathrm{X}^{\prime}$ contains a Hermitian variety which is the union of $q+1$ hyperplanes. The desired results have been obtained.

The only condition $q(x+1)>2(q+1) q^{2}$ implies that

$$
|V|>q^{8}+4 q^{6}+q^{5}-3 q^{4}+4 q^{3}+3 q^{2}+q-1
$$

is required to have these results.

Lemma 3.3 For $\mathrm{X}=\mathrm{H}\left(5, q^{2}\right)$, if $|V|>q^{8}+2 q^{6}+2 q^{5}+2 q^{4}-q^{3}+q+2$, then the case $|L \cap V|=1$ does not occur. 
Proof. Assume that no Hermitian variety in the pencil of Hermitian varieties defined by $\mathrm{X}$ and $\mathrm{X}^{\prime}$ is the union of $q+1$ hyperplanes. Then again there is a singular Hermitian variety $L \mathrm{H}\left(3, q^{2}\right)$ in the pencil and in this Hermitian variety the line $L$ is skew to the solid of $\mathrm{H}\left(3, q^{2}\right)$.

Suppose that we are sure that $x+1$ lines of a cover of size $q^{3}+q$ on $\mathrm{H}\left(3, q^{2}\right)$, as defined in Remark 2.1, define solids through $L$ intersecting $\mathrm{H}\left(5, q^{2}\right)$ in a union of $q+1$ planes. We are sure of this when

$$
1+\frac{|V|-x\left((q+1) q^{4}+q^{2}+1\right)}{q^{3}+q-x}>\left|\mathrm{H}\left(3, q^{2}\right)\right| .
$$

This is equivalent to $|V|>q^{8}+2 q^{6}+q^{5}+q^{4}+q^{3}+x\left(q^{4}-q^{3}+1\right)$.

Similarly as in the preceding proof, for more than $q(x+1)$ lines $\ell$ of the base $H\left(3, q^{2}\right)$ of the cone $L H\left(3, q^{2}\right)$, the solid $\langle L, \ell\rangle$ contains $q+1$ planes of $V$, so of $\mathrm{H}\left(5, q^{2}\right)$; they all pass through the unique intersection point $R$ of $L$ with $\mathrm{H}\left(5, q^{2}\right)$, so they all lie in the tangent hyperplane $T_{R}(\mathrm{X})$ to $\mathrm{X}$ in $R$. Hence, this solid $\langle L, \ell\rangle$, and so in particular the line $\ell$, lies completely in $T_{R}(\mathrm{X})$.

If $x \geq q+2$, then the base $\mathrm{H}\left(3, q^{2}\right)$ of $L \mathrm{H}\left(3, q^{2}\right)$ lies completely in $T_{R}(\mathrm{X})$. But also $L$ lies in $T_{R}(\mathrm{X})$ since $L$ shares only one point with $\mathrm{X}$. However, this implies that $L$ and the base $\mathrm{H}\left(3, q^{2}\right)$ of the cone $\mathrm{LH}\left(3, q^{2}\right)$ share a point, but this is false.

So a contradiction is obtained if $x \geq q+2$, which is valid if

$$
|V|>q^{8}+2 q^{6}+2 q^{5}+2 q^{4}-q^{3}+q+2
$$

Corollary 3.4 Let $\mathrm{X}$ be a non-singular Hermitian variety in $\mathrm{PG}\left(5, q^{2}\right)$, and let $V$ be the intersection of $\mathrm{X}$ with another Hermitian variety $\mathrm{X}^{\prime}$.

If $|V|>q^{8}+4 q^{6}+q^{5}-3 q^{4}+4 q^{3}+3 q^{2}+q-1$, then this intersection $V$ is also the intersection of $\mathrm{X}$ with a Hermitian variety which is the union of $q+1$ four-dimensional spaces.

The lower bound on $|V|$ of Corollary 3.4 is again compared to a result of Kestenband to have an idea of the sharpness of the bound of the preceding corollary.

Theorem 3.5 ([10, Lemma 3]) There exists a pencil of $q+1$ non-singular Hermitian varieties in $\mathrm{PG}\left(5, q^{2}\right)$ intersecting in

$$
\frac{\left(q^{6}-1\right)\left(q^{4}+1\right)}{q^{2}-1}=q^{8}+q^{6}+2 q^{4}+q^{2}+1
$$

points. 


\section{A divisibility condition on the weights}

In this section, it is proven that the weights of the code $C_{H e r m}(\mathrm{X})$ are divisible by $q^{N-1}$ in case $\mathrm{X}$ is a non-singular Hermitian variety in $\mathrm{PG}\left(N, q^{2}\right)$. This result is a particular case of a more general result on the divisibility of the functional codes $C_{h}(\mathrm{X})$, defined on the non-singular Hermitian variety X of $\mathrm{PG}\left(N, q^{2}\right)$ by the hypersurfaces of degree $h$ [3].

To achieve this goal, the known result is first of all mentioned that a Hermitian variety $\mathrm{X}$ in $\mathrm{PG}\left(N, q^{2}\right)$ can be made to correspond to a quadric in $\mathrm{PG}(2 N+1, q)$.

Let X : $\sum_{i, j=0}^{N} a_{i j} X_{i} X_{j}^{q}=0, a_{i j} \in \mathbb{F}_{q^{2}}, a_{i j}^{q}=a_{j i}$.

Define $\mathbb{F}_{q^{2}}$ as a quadratic extension of $\mathbb{F}_{q}$ via an element $e \in \mathbb{F}_{q^{2}} \backslash \mathbb{F}_{q}$, satisfying a quadratic equation $X^{2}-X-b=0$, so $e^{2}=e+b, e^{q}=-e+1$, and $e^{q+1}=-b$.

Then every element $X_{i} \in \mathbb{F}_{q^{2}}$ can be described as $X_{i}=Y_{i}+e Z_{i}, Y_{i}, Z_{i} \in \mathbb{F}_{q}$. Substitute $X_{i}=Y_{i}+e Z_{i}$ in the equation of $\mathrm{X}$. By using the above description for $e^{2}, e^{q}, e^{q+1}$, and using that $Y_{i}^{q}=Y_{i}$ and that $Z_{i}^{q}=Z_{i}$, the following equation in the variables $Y_{i}$ and $Z_{i}$ is obtained:

$$
\begin{aligned}
\mathrm{X}: & \sum_{i=0}^{N}\left(a_{i i} Y_{i}^{2}+a_{i i} Y_{i} Z_{i}-b a_{i i} Z_{i}^{2}\right)+ \\
& \sum_{i, j=0 ; i<j}^{N}\left((2 \alpha+\beta) Y_{i} Y_{j}+(\alpha-2 \beta b) Y_{i} Z_{j}+(\alpha+\beta(2 b+1)) Z_{i} Y_{j}-(2 \alpha+\beta) b Z_{i} Z_{j}\right)=0,
\end{aligned}
$$

which defines a quadric in $\mathrm{PG}(2 N+1, q)$.

Theorem 4.1 For a non-singular Hermitian variety $\mathrm{X}$ in $\mathrm{PG}\left(N, q^{2}\right)$, the weights of the code $C_{H e r m}(\mathrm{X})$ are divisible by $q^{N-1}$.

Proof. In this proof, the theorem of Ax and Katz is used [8, Theorem 1.0].

The intersection points of the Hermitian variety $\mathrm{X}$ in $\mathrm{PG}\left(N, q^{2}\right)$ with another Hermitian variety $\mathrm{X}^{\prime}$ in $\mathrm{PG}\left(N, q^{2}\right)$ correspond to the intersection points of two corresponding quadrics $\mathrm{Q}$ and $\mathrm{Q}^{\prime}$ in $\mathrm{PG}(2 N+1, q)$, or alternatively in the vector space $V(2 N+2, q)$.

In this vector space $V(2 N+2, q)$, in the notation of [8, Theorem 1.0], the number of intersection points is $N(S, T, f) \equiv 0\left(\bmod q^{\mu(S, T, f)}\right)$, where

$$
\mu(S, T, f) \geq \frac{\operatorname{Card}(S)-\sum_{i \in T} d_{i}}{\sup _{i \in T}\left(d_{i}\right)} .
$$

Here $\operatorname{Card}(S)=2 N+2$, since there are $2 N+2$ variables $Y_{i}, Z_{i}, i=0, \ldots, N$, and $d_{1}=d_{2}=2$ since the intersection of two quadrics is investigated.

So

$$
\mu(S, T, f) \geq \frac{2 N+2-4}{2}=N-1 .
$$

Consequently, in $V(2 N+2, q)$, the number of elements in $\mathrm{X} \cap \mathrm{X}^{\prime}$ is $0\left(\bmod q^{N-1}\right)$, and in $\mathrm{PG}(2 N+1, q)$,

$$
\left|\mathrm{X} \cap \mathrm{X}^{\prime}\right|=\frac{k q^{N-1}-1}{q-1},
$$


for some $k \in \mathbb{N}^{*}$.

Rewriting, this is equivalent to

$$
\left|\mathrm{X} \cap \mathrm{X}^{\prime}\right|=\frac{k q^{N-1}-1}{q-1}=k^{\prime} q^{N-1}+\frac{q^{N-1}-1}{q-1},
$$

with $k=k^{\prime}(q-1)+1$, for some $k^{\prime} \in \mathbb{N}$.

So

$$
\left|\mathrm{X} \cap \mathrm{X}^{\prime}\right|=k^{\prime} q^{N-1}+q^{N-2}+q^{N-3}+\cdots+q+1
$$

in $\operatorname{PG}(2 N+1, q)$.

By changing the setting from $\mathrm{PG}\left(N, q^{2}\right)$ to $\mathrm{PG}(2 N+1, q)$, the points of $\mathrm{PG}\left(N, q^{2}\right)$ correspond to the lines of a 1-spread of $\mathrm{PG}(2 N+1, q)$, i.e., a partitioning of the points of $\mathrm{PG}(2 N+1, q)$ into $\left(q^{2 N+2}-1\right) /\left(q^{2}-1\right)$ pairwise disjoint lines.

Consequently, since every intersection point of $\mathrm{X} \cap \mathrm{X}^{\prime}$ in $\mathrm{PG}\left(N, q^{2}\right)$ defines $q+1$ collinear intersection points of one of those lines of this 1-spread of $\mathrm{PG}(2 N+1, q),\left|\mathrm{X} \cap \mathrm{X}^{\prime}\right| \equiv 0$ $(\bmod q+1)$ in the setting of $\mathrm{PG}(2 N+1, q)$.

The theorem of Ax and Katz is now applied to the Hermitian variety $\mathrm{X}$ itself in the setting of $\mathrm{PG}(2 N+1, q)$. This gives $\mu(S, T, f) \geq(2(N+1)-2) / 2=N$. So $|\mathrm{X}| \equiv 0$ $\left(\bmod q^{N}\right)$ in $V(2 N+2, q)$. Hence, over $\mathrm{PG}(2 N+1, q),|\mathrm{X}|=\left(j q^{N}-1\right) /(q-1)=$ $j^{\prime} q^{N}+q^{N-1}+q^{N-2}+\cdots+q+1$, with $j=j^{\prime}(q-1)+1$ for some $j^{\prime} \in \mathbb{N}$.

Case 1. Assume that $N$ is even. Then

$$
k^{\prime} q^{N-1}+q^{N-2}+\cdots+q+1 \equiv 0 \quad(\bmod q+1)
$$

in $\operatorname{PG}(2 N+1, q)$, which implies that

$$
k^{\prime} \equiv 1 \quad(\bmod q+1) .
$$

So $k^{\prime}=k^{\prime \prime}(q+1)+1$, which implies that

$$
\left|\mathrm{X} \cap \mathrm{X}^{\prime}\right|=k^{\prime \prime}(q+1) q^{N-1}+q^{N-1}+q^{N-2}+\cdots+q+1
$$

in $\mathrm{PG}(2 N+1, q)$.

Similarly, in $\mathrm{PG}(2 N+1, q)$,

$$
|\mathrm{X}|=j^{\prime} q^{N}+q^{N-1}+\cdots+q+1 \equiv 0 \quad(\bmod q+1),
$$

which implies that $j^{\prime}=j^{\prime \prime}(q+1)$ for some $j^{\prime \prime} \in \mathbb{N}$.

Then, in $\mathrm{PG}(2 N+1, q)$,

$$
|\mathrm{X}|=j^{\prime \prime}(q+1) q^{N}+q^{N-1}+\cdots+q+1 .
$$

So the weight of a codeword of $C_{H e r m}(\mathrm{X})$ in the setting of $\mathrm{PG}(2 N+1, q)$ is

$$
j^{\prime \prime}(q+1) q^{N}-k^{\prime \prime}(q+1) q^{N-1} \equiv 0 \quad\left(\bmod q^{N-1}\right) .
$$


But one point of $\mathrm{X} \cap \mathrm{X}^{\prime}$ in $\mathrm{PG}\left(N, q^{2}\right)$ corresponds to $q+1$ collinear intersection points of $\mathrm{X} \cap \mathrm{X}^{\prime}$ in $\mathrm{PG}(2 N+1, q)$, so in the setting of $\mathrm{PG}\left(N, q^{2}\right)$, the weight of a codeword of $C_{H e r m}(\mathrm{X})$ is

$$
j^{\prime \prime} q^{N}-k^{\prime \prime} q^{N-1} \equiv 0 \quad\left(\bmod q^{N-1}\right) .
$$

This shows that the weight of this codeword of $C_{H e r m}(\mathrm{X})$ is a multiple of $q^{N-1}$.

Case 2. Assume that $N$ is odd.

This case is treated in the same way as the case $N$ even.

\section{Small weights of $C_{H e r m}(\mathrm{X})$}

The Tables 2 and 6 show that the preceding results determine the 4 smallest weights of $C_{\text {Herm }}(\mathrm{X})$. These small weight codewords correspond to the intersection of the nonsingular Hermitian variety $\mathrm{X}$ in $\mathrm{PG}\left(N, q^{2}\right)$ with Hermitian varieties $\mathrm{X}^{\prime}$ which are the union of $q+1$ hyperplanes. These latter $q+1$ hyperplanes have an $(N-2)$-dimensional space $\Pi_{N-2}$ in common. The polar space of $\Pi_{N-2}$ with respect to $\mathrm{X}$ is a line $L$, which can be tangent, secant to, or contained in X. The discussion is made depending on the position of $L$ with respect to the Hermitian variety X.

If $L$ is secant to X, then $\Pi_{N-2}$ intersects $\mathrm{X}$ in a non-singular Hermitian variety $H_{N-2}$ in $\mathrm{PG}\left(N-2, q^{2}\right)$. If $P \in L \cap \mathrm{X}$, then the hyperplane $\left\langle P, \Pi_{N-2}\right\rangle$ intersects $\mathrm{X}$ in the tangent cone $P \mathrm{H}_{N-2}$. If $P \in L \backslash \mathrm{X}$, then the hyperplane $\left\langle P, \Pi_{N-2}\right\rangle$ intersects $\mathrm{X}$ in a non-singular Hermitian variety $\mathrm{H}_{N-1}$ in $\mathrm{PG}\left(N-1, q^{2}\right)$. Since two distinct Baer sublines share 0,1 , or 2 points, consequently, $q+1,0,2$ or one of the $q+1$ hyperplanes of $\mathrm{X}^{\prime}$ through $\Pi_{N-2}$ can contain a point of $L \cap \mathrm{X}$, respectively cases (1),(2),(3) and (4) in Table 2. In the case that $L$ is tangent to $\mathrm{X}$, then $\Pi_{N-2}$ intersects $\mathrm{X}$ in a singular Hermitian variety $\mathrm{PH}_{N-3}$ in $\mathrm{PG}\left(N-2, q^{2}\right)$. In this case, one or none of the $q+1$ hyperplanes of $\mathrm{X}^{\prime}$ through $\Pi_{N-2}$ can contain the intersection point of $L$ with X, respectively cases (6) and (7) in Table 2 . In the case that $L$ is contained in $\mathrm{X}$, then $\Pi_{N-2}$ intersects $\mathrm{X}$ in a singular Hermitian variety $L H_{N-4}$ in $\mathrm{PG}\left(N-2, q^{2}\right)$. In this final case, all the $q+1$ hyperplanes of $\mathrm{X}^{\prime}$ are tangent hyperplanes to $\mathrm{X}$; this is case (5) in Table 2 . In Table $2, H_{i}$ denotes a non-singular Hermitian variety in $\mathrm{PG}\left(i, q^{2}\right), P H_{N-2}$ denotes a singular Hermitian variety in $\mathrm{PG}\left(N-1, q^{2}\right)$ with vertex a point $P$ and base $H_{N-2}, L_{N-4}$ denotes a singular Hermitian variety in $\operatorname{PG}\left(N-2, q^{2}\right)$ with vertex the line $L$ and base $H_{N-4}$, and $P H_{N-3}$ denotes a singular Hermitian variety in $\operatorname{PG}\left(N-2, q^{2}\right)$ with vertex the point $P$ and base $H_{N-3}$. 


\begin{tabular}{|c|c|c|}
\hline & $\Pi_{N-2} \cap \mathrm{X}$ & $\left|\mathrm{X} \cap \mathrm{X}^{\prime}\right|$ \\
\hline$(1)$ & $H_{N-2}$ & $(q+1)\left|P H_{N-2}\right|-q\left|H_{N-2}\right|$ \\
\hline$(2)$ & $H_{N-2}$ & $(q+1)\left|H_{N-1}\right|-q\left|H_{N-2}\right|$ \\
\hline$(3)$ & $H_{N-2}$ & $2\left|P H_{N-2}\right|+(q-1)\left|H_{N-1}\right|-q\left|H_{N-2}\right|$ \\
\hline$(4)$ & $H_{N-2}$ & $\left|P H_{N-2}\right|+q\left|H_{N-1}\right|-q\left|H_{N-2}\right|$ \\
\hline$(5)$ & $L H_{N-4}$ & $(q+1)\left|P H_{N-2}\right|-q\left|L H_{N-4}\right|$ \\
\hline$(6)$ & $P H_{N-3}$ & $(q+1)\left|H_{N-1}\right|-q\left|P H_{N-3}\right|$ \\
\hline$(7)$ & $P H_{N-3}$ & \\
\hline
\end{tabular}

Table 2: Different cases for $\Pi_{N-2} \cap \mathrm{X}$ and the corresponding sizes for $\left|\mathrm{X} \cap \mathrm{X}^{\prime}\right|$.

\section{$5.1 \quad N$ even}

For $N$ even, Table 3 gives for the corresponding intersections of Table 2 the sizes of these intersections. Then Table 4 gives the corresponding weights in the code $C_{H e r m}(\mathrm{X})$. Note that (2) gives the smallest weight $w_{1},(4)$ and (7) give the second smallest weight $w_{1}+q^{N-1}$, cases (3), (5), and (6) give the third smallest weight $w_{1}+2 q^{N-1}$, while case (1) gives the fourth smallest weight $w_{1}+q^{N-1}(q+1)$. Table 4 also gives the number of codewords having these weights. When there are different cases leading to the same weight, in the rightmost column of Table 4, the total number of codewords of that weight is written as a sum of the corresponding numbers of codewords corresponding to the respective cases of Table 2 .

\begin{tabular}{|c|c|}
\hline & $\left|\mathrm{X} \cap \mathrm{X}^{\prime}\right|$ \\
\hline$(1)$ & $q^{2 N-2}+q^{2 N-3}+q^{2 N-5}+\cdots+q^{N+1}-q^{N}+q^{N-1}+q^{N-2}+q^{N-4}+\cdots+q^{2}+1$ \\
\hline$(2)$ & $q^{2 N-2}+q^{2 N-3}+q^{2 N-5}+\cdots+q^{N+1}+2 q^{N-1}+q^{N-2}+q^{N-4}+\cdots+q^{2}+1$ \\
\hline$(3)$ & $q^{2 N-2}+q^{2 N-3}+q^{2 N-5}+\cdots+q^{N+1}+q^{N-2}+q^{N-4}+\cdots+q^{2}+1$ \\
\hline$(4)$ & $q^{2 N-2}+q^{2 N-3}+q^{2 N-5}+\cdots+q^{N-1}+q^{N-2}+q^{N-4}+\cdots+q^{2}+1$ \\
\hline$(5)$ & $q^{2 N-2}+q^{2 N-3}+q^{2 N-5}+\cdots+q^{N+1}+q^{N-2}+q^{N-4}+\cdots+q^{2}+1$ \\
\hline$(6)$ & $q^{2 N-2}+q^{2 N-3}+q^{2 N-5}+\cdots+q^{N+1}+q^{N-2}+q^{N-4}+\cdots+q^{2}+1$ \\
\hline$(7)$ & $q^{2 N-2}+q^{2 N-3}+q^{2 N-5}+\cdots+q^{N-1}+q^{N-2}+q^{N-4}+\cdots+q^{2}+1$ \\
\hline
\end{tabular}

Table 3: Sizes of $\left|\mathrm{X} \cap \mathrm{X}^{\prime}\right|$ corresponding to Table 2. 


\begin{tabular}{|c|c|c|}
\hline & Weight & Number of codewords \\
\hline$(2)$ & $w_{1}=q^{N-1}\left(q^{N}-q^{N-1}-2\right)$ & $\frac{\left(q^{N+1}+1\right)\left(q^{N}-1\right) q^{2 N-1}(q-1)(q-2)}{2(q+1)^{2}}$ \\
\hline$(4)+(7)$ & $w_{2}=w_{1}+q^{N-1}$ & $\frac{\left(q^{N+1}+1\right)\left(q^{N}-1\right) q^{2 N-2}(q-1)}{q+1}+$ \\
& & $\frac{\left(q^{N+1}+1\right)\left(q^{N}-1\right) q^{N}\left(q^{N-1}+1\right)(q-1)}{(q+1)^{2}}$ \\
\hline$(3)+(5)+(6)$ & $w_{3}=w_{1}+2 q^{N-1}$ & $\frac{\left(q^{N+1}+1\right)\left(q^{N}-1\right) q^{2 N}}{2(q+1)}+$ \\
& & $\frac{q\left(q^{N+1}+1\right)\left(q^{N}-1\right)\left(q^{N-1}+1\right)\left(q^{N-2}-1\right)}{\left(q^{2}-1\right)(q+1)}+$ \\
& & $\frac{\left(q^{N+1}+q^{N}-1\right) q^{N-1}\left(q^{N-1}+1\right)}{q+1}$ \\
\hline$(1)$ & $w_{4}=w_{1}+q^{N-1}(q+1)$ & $\frac{\left(q^{N+1}+1\right)\left(q^{N}-1\right) q^{2 N-2}}{(q+1)^{2}}$ \\
\hline
\end{tabular}

Table 4: The four smallest weights of $C_{H e r m}(\mathrm{X}), N$ even.

\section{$5.2 \quad N$ odd}

For $N$ odd, Table 5 gives for the corresponding intersections of Table 2 the sizes of these intersections. Then Table 6 gives the corresponding weights in the code $C_{H e r m}(\mathrm{X})$. Note that (1) gives the smallest weight $w_{1},(3),(5)$, and (6) give the second smallest weight $w_{1}+q^{N}-q^{N-1}$, cases (4) and (7) give the third smallest weight $w_{1}+q^{N}$, while case (2) gives the fourth smallest weight $w_{1}+q^{N-1}(q+1)$. Table 6 also gives the number of codewords having these weights. When there are different cases leading to the same weight, in the rightmost column of Table 6 , the total number of codewords of that weight is written as a sum of the corresponding numbers of codewords corresponding to the respective cases of Table 2 .

\begin{tabular}{|c|c|}
\hline & $\left|\mathrm{X} \cap \mathrm{X}^{\prime}\right|$ \\
\hline$(1)$ & $q^{2 N-2}+q^{2 N-3}+q^{2 N-5}+\cdots+q^{N+2}+2 q^{N}+q^{N-3}+q^{N-5}+\cdots+q^{2}+1$ \\
\hline$(2)$ & $q^{2 N-2}+q^{2 N-3}+q^{2 N-5}+\cdots+q^{N}-q^{N-1}+q^{N-3}+q^{N-5}+\cdots+q^{2}+1$ \\
\hline$(3)$ & $q^{2 N-2}+q^{2 N-3}+q^{2 N-5}+\cdots+q^{N}+q^{N-1}+q^{N-3}+\cdots+q^{2}+1$ \\
\hline$(4)$ & $q^{2 N-2}+q^{2 N-3}+q^{2 N-5}+\cdots+q^{N}+q^{N-3}+q^{N-5}+\cdots+q^{2}+1$ \\
\hline$(5)$ & $q^{2 N-2}+q^{2 N-3}+q^{2 N-5}+\cdots+q^{N}+q^{N-1}+q^{N-3}+\cdots+q^{2}+1$ \\
\hline$(6)$ & $q^{2 N-2}+q^{2 N-3}+q^{2 N-5}+\cdots+q^{N}+q^{N-1}+q^{N-3}+\cdots+q^{2}+1$ \\
\hline$(7)$ & $q^{2 N-2}+q^{2 N-3}+q^{2 N-5}+\cdots+q^{N}+q^{N-3}+q^{N-5}+\cdots+q^{2}+1$ \\
\hline
\end{tabular}

Table 5: Sizes of $\left|\mathrm{X} \cap \mathrm{X}^{\prime}\right|$ corresponding to Table 2. 


\begin{tabular}{|c|c|c|}
\hline & Weight & Number of codewords \\
\hline$(1)$ & $w_{1}=q^{N-1}\left(q^{N-1}-1\right)(q-1)$ & $\frac{\left(q^{N+1}-1\right)\left(q^{N}+1\right) q^{2 N-2}}{(q+1)^{2}}$ \\
\hline$(3)+(5)+(6)$ & $w_{2}=w_{1}+q^{N}-q^{N-1}$ & $\frac{\left(q^{N+1}-1\right)\left(q^{N}+1\right) q^{2 N}}{2(q+1)}$ \\
& & $\frac{q\left(q^{N+1}-1\right)\left(q^{N}+1\right)\left(q^{N-1}-1\right)\left(q^{N-2}+1\right)}{N\left(q^{2}-1\right)(q+1)}+$ \\
& & $\frac{q^{N-1}\left(q^{N+1}-1\right)\left(q^{N}+1\right)\left(q^{N-1}-1\right)}{q+1}$ \\
\hline$(4)+(7)$ & $w_{3}=w_{1}+q^{N}$ & $\frac{\left(q^{N+1}-1\right)\left(q^{N}+1\right) q^{2 N-2}(q-1)}{q+1}+$ \\
& & $\frac{q^{N}\left(q^{N+1}-1\right)\left(q^{N}+1\right)\left(q^{N-1}-1\right)(q-1)}{(q+1)^{2}}$ \\
\hline$(2)$ & $w_{4}=w_{1}+q^{N-1}(q+1)$ & $\frac{q^{2 N-1}\left(q^{N+1}-1\right)\left(q^{N}+1\right)(q-1)(q-2)}{2(q+1)^{2}}$ \\
\hline
\end{tabular}

Table 6: The four smallest weights of $C_{H e r m}(\mathrm{X}), N$ odd.

\section{Acknowledgments}

The authors would like to thank the two anonymous referees for their remarks and their comments on the preliminary version of their paper.

\section{References}

[1] F.A.B. Edoukou, Codes correcteurs d'erreurs construits à partir des variétés algébriques, PhD Thesis, Université de la Méditerranée (Aix-Marseille II), France, 2007.

[2] F.A.B. Edoukou, Codes defined by forms of degree 2 on quadric surfaces, IEEE Trans. Inform. Theory 54 (2), (2008), 860-864.

[3] F.A.B. Edoukou, S. Ling, and C. Xing, New informations on the structure of the functional codes defined by forms of degree $h$ on non-degenerate Hermitian varieties in $\mathbb{P}^{n}\left(\mathbb{F}_{q}\right)$. arXiv: 090\%.4548, under submission.

[4] F.A.B. Edoukou, A. Hallez, F. Rodier, and L. Storme, On the small weight codewords of the functional codes $C_{2}(Q), Q$ a non-singular quadric. J. Pure Applied Algebra. In Press. Online publication complete: 6-JAN-2010. DOI information:10.1016/j.jpaa.2009.12.017.

[5] A. Hallez and L. Storme, Functional codes arising from quadric intersections with Hermitian varieties. Finite Fields Appl. 16 (2010), 27-35.

[6] J.W.P. Hirschfeld, Projective Geometries over Finite Fields (Second Edition), Oxford University Press, Oxford, 1998.

[7] J.W.P. Hirschfeld and J.A. Thas, General Galois Geometries. Oxford Mathematical Monographs. Oxford University Press, 1991.

[8] N.M. Katz, On a Theorem of Ax, Amer. J. Math. 93 (1971), no. 2, 485-499. 
[9] B.C. Kestenband, Projective geometries that are disjoint unions of caps. Canad. J. Math. 32 (1980), no. 6, 1299-1305.

[10] B.C. Kestenband, Hermitian configurations in odd-dimensional projective geometries. Canad. J. Math. 33 (1981), no. 2, 500-512.

[11] G. Lachaud, Number of points of plane sections and linear codes defined on algebraic varieties. In Arithmetic, Geometry, and Coding Theory. (Luminy, France, 1993), Walter De Gruyter, Berlin-New York, (1996), 77-104.

[12] K. Metsch, The sets closest to ovoids in $Q^{-}(2 n+1, q)$. Finite geometry and combinatorics (Deinze, 1997). Bull. Belg. Math. Soc. Simon Stevin 5 (1998), 389-392.

Address of the authors:

F.A.B. Edoukou, Division of Mathematical Sciences, School of Physical and Mathematical Sciences, Nanyang Technological University, SPMS-04-01, 21 Nanyang Link, Singapore 637371 (abfedoukou@ntu.edu.sg)

F. Rodier, CNRS, Institut de Mathématiques de Luminy, Luminy case 907, 13288 Marseille cedex 9, France (rodier@iml.univ-mrs.fr)

A. Hallez and L. Storme, Department of pure mathematics and computer algebra, Ghent University, Krijgslaan 281-S22, 9000 Ghent, Belgium. (athallez@cage.ugent.be, L. Storme: ls@cage.ugent.be, http://cage.ugent.be/ ls) 